\title{
Prevalença d'estrès i factors associats al Grau en Psicologia
}

\section{Prevalencia de estrés y factores asociados en el Grado en Psicología}

\author{
Alejandro Viciano-Molina \\ al290692@uji.es \\ Rafael Ballester-Arnal \\ rballest@uji.es
}

\section{Resum}

Introducció: Es coneix el paper de l'estrès en la gènesi de trastorns mentals i físics, la seua repercussió sobre el rendiment acadèmic, en l'inici i empitjorament del consum patològic de substàncies i, així i tot, no ha estat pràcticament estudiat en l'estudiantat de Psicologia.

Mètode: S'administrà una bateria de qüestionaris d'autoinforme a 238 estudiants de grau de la Facultat de Ciències de la Salut (Psicologia, Infermeria i Medicina) de la Universitat Jaume I de Castelló sobre estrès percebut, estressors i característiques sociodemogràfiques.

Resultats: La puntuació mitjana d'estrès percebut en cada títol va ser 26,15 en Infermeria; 27,01 en Medicina i 25,85 en Psicologia sense diferències estadísticament significatives entre carreres $(p=0,662)$. En Psicologia hi ha un $57,31 \%$ d'estudiantes amb estrès moderat i un 10,97 \% amb alt estrès, sent els estressors més freqüents perdre el control sobre el propi horari $(66,7 \%)$, dificultats econòmiques $(60,7 \%)$, problemes de parella $(50 \%)$ i la malaltia greu d'un ésser estimat $(46,4 \%)$. L'estudiantat amb menors puntuacions en estrès informa una major salut física $(F=5,77 ; p=0,016)$ i mental $(F=13,95$; $p=0,001)$ percebudes. No hi han diferències quant a l'estrès en funció d'altres variables (gènere, origen, estat civil, curs, situació laboral, assignatures pendents, malaltia física i orientació sexual), excepte per a patir malaltia mental de base $(t=2,06 ; p=0,042)$ i insatisfacció $a m b$ la imatge corporal $(F=4,03 ; p=0,010)$.

Discussió: Més del $70 \%$ de l'alumnat de Psicologia pateix estrès moderat-alt. Es deuen realitzar intervencions preventives tant en l'estudiantat ja afectat per nivells elevats d'estrès com en el que comença a experimentar-lo, per dotar-lo d'eines d'afrontament efectives i prevenir possibles problemes derivats.

Paraules clau: estrès, estudiants, psicologia, salut percebuda. 


\section{Abstract}

Introduction: It is well known that stress plays a role in the genesis of mental and physical disorders. Its implications have great importance, since it can impact academic performance and trigger the beginning and worsening of pathological substance consumption. Despite this, its impact has been scarcely studied in Psychology students.

Methods: A battery of self-report questionnaires was given to 238 degree students from the Faculty of Health Sciences (Psychology, Nursing and Medicine) on perceived stress, stressors and sociodemographic characteristics.

Results: The average score of perceived stress in each title was 26.15 in Nursing; 27.01 in Medicine and 25,85 in Psychology without statistically significant differences between degrees $(p=0.662)$. In Psychology there is a $57.31 \%$ of students with moderate stress and a $10.97 \%$ with high stress, being the most frequent stressors losing control over their own schedule $(66.7 \%)$, economic difficulties $(60.7 \%)$, relationship problems $(50 \%)$ and serious illness of a loved one $(46.4 \%)$. Students with the lowest stress scores reported the highest perceived physical $(F=5.77 ; p=0.016)$ and mental $(F=13.95 ; p=0.001)$ health. No differences were found regarding stress in relation to other variables (gender, origin, civil status, course, work situation, pending subjects, physical illness and sexual orientation), except for suffering a mental illness $(t=2.06 ; p=0.042)$ and dissatisfaction with own body image $(F=4.03 ; p=0.010)$.

Discussion: Over $70 \%$ of psychology students suffer moderate-high stress. Preventive interventions should be carried out, both for students already affected by high levels of stress and on individuals that begin to experience it, in order to provide effective coping tools and prevent potential problems.

Keywords: stress, students, psychology, perceived health.

\section{Resumen}

Introducción: Se conoce el papel del estrés en la génesis de trastornos mentales y físicos, su repercusión sobre el rendimiento académico, en el inicio y empeoramiento del consumo patológico de sustancias y, aun así, no ha sido prácticamente estudiado en el estudiantado de Psicología.

Método: Se administró una batería de cuestionarios de autoinforme a 238 estudiantes de grado de la Facultad de Ciencias de la Salud (Psicología, Enfermería y Medicina) de la Universitat Jaume I de Castellón sobre estrés percibido, estresores y características sociodemográficas.

Resultados: La puntuación media de estrés percibido en cada título fue 26,15 en Enfermería; 27,01 en Medicina y 25,85 en Psicología sin diferencias estadísticamente significativas entre carreras $(p=0,662)$. En Psicología hay un $57,31 \%$ de estudiantes con estrés moderado y un 10,97\% con alto estrés, siendo los estresores más frecuentes perder el control sobre el propio horario $(66,7 \%)$, dificultades económicas $(60,7 \%)$, problemas de pareja $(50 \%)$ y la enfermedad grave de un ser querido $(46,4 \%)$. El estudiantado con menores puntuaciones en estrés informa una mayor salud física $(F=5,77 ; p=0,016)$ y mental $(F=13,95 ; p=0,001)$ percibidas. No hay diferencias en cuanto al estrés en función de otras variables (género, origen, estado civil, curso, situación laboral, asignaturas pendientes, enfermedad física y orientación sexual), excepto para padecer enfermedad mental de base $(t=2,06 ; p=0,042)$ e insatisfacción con la imagen corporal $(F=4,03 ; p=0,010)$.

Discusión: Más del $70 \%$ del alumnado de Psicología sufre estrés moderadoalto. Se deben realizar intervenciones preventivas tanto en el estudiantado ya 
afectado por niveles elevados de estrés como en el que comienza a experimentarlo, para dotarlo de herramientas de afrontamiento efectivas y prevenir posibles problemas derivados.

Palabras clave: estrés, estudiantes, psicología, salud percibida.

\section{Introducció}

L'educació superior és una etapa d'enriquiment dels coneixements i de desenvolupament personal i professional per a les persones que hi poden accedir. No obstant això es pot apreciar que té un preu més enllà de les taxes universitàries i de matricula i és que l'estrès forma part de la vida diària de una gran proporció d'estudiantat universitari (Pierceall i Keim 2007).

Pel que fa a l'estudiantat de ciències de la salut, l'estrès ha estat poc estudiat i, quan ho ha sigut, s'ha centrat fonamentalment en estudiantat de Medicina (Dahlin, Joneborg i Runeson 2005; Goel, Akarte, Agrawal i Yadav 2016; Guthrie et al. 1998; Guthrie et al. 1997; Kötter, Wagner, Brüheim i Voltmer 2017; Matheson et al. 2016; Sheets, Gorenflo i Forney 1993; Youssef 2016) i més recentment en el d'Infermeria (Admi, Moshe-Eilon, Sharon i Mann 2018; Suárez-García, Maestro-González, Zuazua-Rico, Sánchez-Zaballos i Mosteiro-Díaz 2018; Valero-Chillerón et al. 2019; Zupiria-Gorostidi, Uranga-Iturriotz, Alberdi-Erize i Barandiaran-Lasa 2003).

Aquests estudis relacionen l'estrès amb tindre menor seguretat en assolir les metes acadèmiques (Pierceall i Keim 2007), a haver passat més temps a la titulació (Youssef 2016) i generalment amb el gènere femení (Bore, Kelly i Nair 2016; Pierceall i Keim 2007; Suárez-García, Maestro-González, Zuazua-Rico, SánchezZaballos i Mosteiro-Díaz 2018). A més descriuen que els principals estressors són: en Medicina la falta de temps lliure, no poder influir en el currículum acadèmic, que no hi haja un paper clar per a l'estudiantat, la càrrega de treball, l'estudi i els exàmens, la seva situació financera... (Bacchi i Licinio 2017; Dahlin i Runeson 2007; Guthrie et al. 1997; Matheson et al. 2016) i en Infermeria percebre conflicte entre el que s'ha après i la realitat, el dolor i el sofriment dels pacients, percebre un coneixement i capacitació inadequats, la percepció de recursos hospitalaris insuficients (Admi, Moshe-Eilon, Sharon i Mann 2018), també fer malament la seva feina i perjudicar al pacient, barrejar medicaments i punxar-se amb una agulla infectada (Suárez-García, Maestro-González, Zuazua-Rico, Sánchez-Zaballos i Mosteiro-Díaz 2018; Valero-Chillerón et al. 2019).

Donada la falta d'estudis recents sobre la presència d'aquesta problemàtica i els factors que s'hi relacionen a l'estudiantat de Psicologia, els objectius del treball van ser:

1. Establir la prevalença d'estrès problemàtic a l'estudiantat de Psicologia i comparar el nivell d'estrès amb les altres titulacions de la Facultat de Ciències de la Salut de la Universitat Jaume I (UJI).

2. Identificar els principals estressors a l'alumnat.

3. Establir què factors es relacionen amb alts nivells d'estrès. 


\section{Mètode}

\section{Participants}

A la taula 1 es pot observar com, de forma similar a la mostra total $(79,80 \%)$, la major part de les persones del Grau en Psicologia enquestades van ser dones $(77,40 \%)$, amb una edat mitjana de 23,09 anys i també majoritàriament d'origen espanyol $(89,30 \%)$ encara que amb orígens més diversos que en la mostra total. Les situacions més comuns van ser: estar casat/da o tindre parella estable $(66,70 \%$ en Psicologia i $67,60 \%$ en tota la mostra), no compaginar estudis amb treball $(75 \%$ en Psicologia i $82,40 \%$ en tota la mostra) i no tindre assignatures d'altres cursos $(73,80 \%$ en Psicologia i $79,80 \%$ en tota la mostra). Finalment, s'aprecia una major presència d'alumnat del primer curs, tant a la mostra total $(29,40 \%)$ com a la de Psicologia $(32,10 \%)$, que dels altres cursos.

Taula 1

Característiques de la mostra del Grau en Psicologia i total

\begin{tabular}{|c|c|c|}
\hline & Psicologia & Total \\
\hline \multicolumn{3}{|l|}{ Gènere $(\%)$} \\
\hline Dona & $77,40 \%$ & $79,80 \%$ \\
\hline Home & $22,60 \%$ & $20,20 \%$ \\
\hline Edat mitjana (DT) & $23,09(6,52)$ & $22,37(5,26)$ \\
\hline \multicolumn{3}{|l|}{ Origen $(\%)$} \\
\hline Espanyol & $89,30 \%$ & $92,00 \%$ \\
\hline Europeu (no espanyol) & $6,00 \%$ & $3,40 \%$ \\
\hline Llatinoamericà & $4,80 \%$ & $2,90 \%$ \\
\hline Asiàtic & $0,00 \%$ & $1,30 \%$ \\
\hline Anglosaxó & $0,00 \%$ & $0,40 \%$ \\
\hline Africà & $0,00 \%$ & $0,00 \%$ \\
\hline \multicolumn{3}{|l|}{ Estat civil (\%) } \\
\hline Fadrí/na o sense parella estable & $33,30 \%$ & $32,40 \%$ \\
\hline Casat /a o parella estable & $66,70 \%$ & $67,60 \%$ \\
\hline \multicolumn{3}{|l|}{ Curs $(\%)$} \\
\hline 1r & $32,10 \%$ & $29,40 \%$ \\
\hline $2 n$ & $17,90 \%$ & $16,40 \%$ \\
\hline $3 r$ & $22,60 \%$ & $18,90 \%$ \\
\hline $4 \mathrm{t}$ & $27,40 \%$ & $21,00 \%$ \\
\hline 5 é & $0,00 \%$ & $7,10 \%$ \\
\hline 6é & $0,00 \%$ & $7,10 \%$ \\
\hline \multicolumn{3}{|l|}{ Situació laboral (\%) } \\
\hline Sols estudien & $75,00 \%$ & $82,40 \%$ \\
\hline TC i EC & $4,80 \%$ & $2,10 \%$ \\
\hline TC i EP & $1,20 \%$ & $1,30 \%$ \\
\hline TM i EC & $16,70 \%$ & $12,60 \%$ \\
\hline TM i EP & $2,40 \%$ & $1,70 \%$ \\
\hline \multicolumn{3}{|l|}{ Assignatures pendents (\%) } \\
\hline No & $73,80 \%$ & $79,40 \%$ \\
\hline Sí, per suspens & $23,80 \%$ & $16,40 \%$ \\
\hline Sí, per trasllat & $2,40 \%$ & $4,20 \%$ \\
\hline Mitja acadèmica (DT) & $7,41(0,85)$ & $7,65(0,77)$ \\
\hline
\end{tabular}

Nota: TC, treball jornada completa; TM, treball mitja jornada; EC, estudis temps complet; EP, estudis temps parcial

${ }^{*} \mathrm{p} \leq 0,05 ;{ }^{* *} \mathrm{p} \leq 0,01 ; * * * \mathrm{p} \leq 0,001$ 


\section{Instruments}

Es va mesurar l'estrès percebut mitjançant la versió espanyola de la Perceived Stress Scale versió de 14 ítems (PSS14) de Cohen, Kamarck i Mermelstein (1983), una escala d'estrès percebut de 14 ítems tipus Likert que mesuren la freqüència d'impredictibilitat, sobrecàrrega i incontrolabilitat percebudes en l'últim mes i dona una puntuació que pot anar de 1 a 56. L'aproximació dels nivells d'estrès és la següent: menys de 20 és baix estrès, de 20 a 36 és estrès moderat i més de 36 equival a estrès elevat.

El estressors van ser recollits a través d'una escala dissenyada ad hoc. Constava de 14 ítems per avaluar la vivència d'estressors concrets i la seva repercussió en la vida de l'estudiantat. Les possibles respostes comprenien: «No m'ha passat»; «Em va passar i no em va afectar gens»; "Em va passar i em va afectar una mica»; «Em va passar i em va afectar bastant»; «Em va passar i em va afectar molt». Alguns exemples són patir problemes acadèmics importants, dificultats econòmiques, haver patit una malaltia greu, accident o ingrés hospitalari, entre d'altres.

Les característiques sociodemogràfiques i de salut autoinformades també es van obtindre mitjançant preguntes dissenyades ad hoc. Es va preguntar per: gènere, origen, estat civil, curs, situació laboral, assignatures pendents, estat de salut físic i mental, malaltia física o mental diagnosticada, interferència de les malalties físiques i mentals, orientació sexual i satisfacció amb la imatge corporal.

\section{Procediment}

Per tal d'aconseguir els objectius proposats, es va calcular la mida mostral necessària, que es va estimar en 227 persones, tenint en compte la població total de 1505 estudiants, un nivell de confiança del $95 \%$, la referència en l'estimació dels càlculs del $50 \%$ i un error mostral màxim del $6 \%$. La mostra total va quedar conformada per 89 estudiants de Medicina, 84 de Psicologia i 65 d'Infermeria.

Seguint un mostreig no probabilístic intencional, es va contactar mitjançant correu electrònic amb l'estudiantat matriculat en estudis de grau de la Facultat de Ciències de la Salut de I'UJI. El correu incloïa un text informatiu sobre la investigació i el vincle a la plataforma en línia, on es recollia el consentiment informat $i$, una vegada llegit i acceptat aquest, es podien respondre els qüestionaris d'avaluació. Es van fer recordatoris periòdicament.

\section{Análisis de variables}

Es va utilitzar el programari IBM SPSS Statistics (versió 23) per executar les proves estadístiques. Es van calcular estadístics descriptius (mitges, desviacions estàndard i freqüències) per reflectir la prevalença de nivells problemàtics d'estrès, juntament amb les fonts d'estrès.

Es va analitzar la relació de l'estrès percebut amb les variables descriptives clau, incloent el gènere, l'origen, l'estat civil, el curs, la situació laboral, la presència d'assignatures pendents, l'estat de salut físic i mental, la malaltia física o mental diagnosticada, la interferència de les possibles malalties físiques i mentals, l'orientació sexual i la satisfacció amb la imatge corporal. Es van utilitzar les proves $\mathrm{t}$ de Student i ANOVA, que comparen les puntuacions en les variables dependents 
entre els 2 o més grups categorials. Totes les proves van ser bilaterals amb tasses d'error tipus I de comparació de 0,05.

\section{Resultats}

Les puntuacions mitjanes de cada titulació, així com la proporció d'alumnat dins de cada nivell d'estrès segons els criteris del PSS14, es representen a la taula 2. La puntuació mitjana en estrès d'Infermeria $(26,15)$, Medicina $(27,01)$ i Psicologia $(25,85)$ es situa en el nivell moderat, encara que amb una variabilitat considerable en tots tres casos. Així, s'observa que la prevalença d'estrès moderat és del $60,71 \%$ i la d'estrès elevat del $11,90 \%$ en Psicologia, sent similar a les de les altres titulacions, al no donar-se diferències estadísticament significatives $\left(X^{2}=6,64\right.$; $p=0,662)$.

Taula 2

Prevalença d'estrès a les titulacions de la Facultat de Ciències de la Salut

\begin{tabular}{lcccccc}
\hline \multicolumn{1}{c}{ Grau } & & \multicolumn{5}{c}{ PSS14 } \\
\hline & Total mitja (DT) & F & $<20$ & $20-36$ & $>36$ & $X^{2}$ \\
Infermeria & $26,15(7,59)$ & 0,413 & $21,50 \%$ & $72,31 \%$ & $6,15 \%$ & 6,64 \\
Medicina & $27,01(9,56)$ & & $27 \%$ & $55,06 \%$ & $17,98 \%$ & \\
Psicologia & $25,85(8,59)$ & & $27,40 \%$ & $60,71 \%$ & $11,90 \%$ & \\
\hline
\end{tabular}

Nota: ${ }^{*} p \leq 0,05 ;{ }^{* *} p \leq 0,01 ;{ }^{* * *} p \leq 0,001$

A la figura 1 es representa el percentatge d'alumnes de Psicologia que han experimentat cadascun dels estressors. Es pot veure com els mes freqüents són sentir que no es té el control del propi horari $(66,70 \%)$, tindre dificultats econòmiques $(60,70 \%)$ i haver tingut problemes de parella $(50 \%)$, quedant altres com la malaltia greu d'un ésser estimat, els problemes acadèmics i socials o la mort d'un ésser estimat, entre altres, per baix del $50 \%$ d'ocurrència.

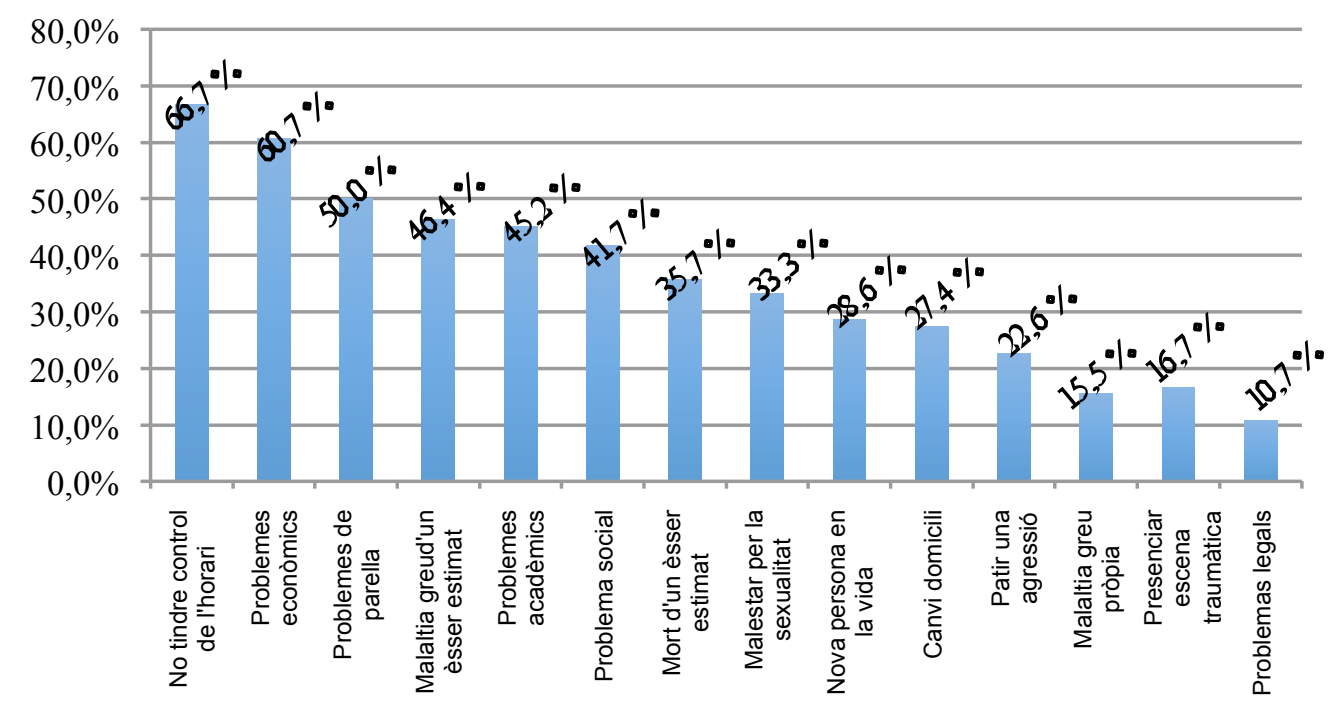

Figura 1. Freqüència d'estressors en l'alumnat del Grau en Psicologia. 
Quant a les diferents puntuacions en l'estrès percebut que es donen en funció de les variables demogràfiques i acadèmiques, es pot observar a la taula $3 \mathrm{com}$ la puntuació dels homes és lleugerament superior a la de les dones (26,44 i 25,66 respectivament), encara que no de forma estadísticament significativa.

Tampoc s'han donat aquestes diferències quant a l'origen, tot i que les persones llatinoamericanes tenen de mitja 33 punts i les d'espanyoles i europees són respectivament de 25,34 i 24,6 .

Respecte a l'estat civil o situació de parella, la mitja de les persones fadrines o sense parella estable $(26,32)$ no ha estat diferent de forma estadísticament significativa que la de les persones casades o amb parella estable $(24,81)$.

L'estrès, encara que no de forma estadísticament significativa ( $F=0,479 ; p=0,698)$, ha sigut major al segon curs amb una mitja de 25,47 i al tercer curs amb 27,89 punts, que en primer i quart on les mitges se situen en 24,84 i 24,87 respectivament.

La situació laboral tampoc ha influït en obtindré majors o menors puntuacions $(\mathrm{t}=0,556$; $p=0,234$ ) tot i que les mitges són de 26,58 per a les persones que només estudien $i$ 21,5 per a les que compaginen treball amb estudis.

Pel que fa a la presència d'assignatures d'altres cursos, la puntuació mitjana en estrès de les persones que no en tenen és de 24,85, menor als 28,05 de les que en tenen per suspensos i els 26,5 de les que en tenen per trasllat d'expedient. No s'hi van trobar diferències estadísticament significatives.

Taula 3

Diferències d'estrès segons variables demogràfiques i acadèmiques

\begin{tabular}{|c|c|c|c|}
\hline Variable & $\begin{array}{c}\text { PSS14 } \\
\text { Mitja (DT) }\end{array}$ & t Student & $\mathrm{F}$ \\
\hline Gènere & & 0,338 & \\
\hline Home & $26,44(7,92)$ & & \\
\hline Dona & $25,66(8,88)$ & & \\
\hline Origen & & & 1,579 \\
\hline Espanyol & $25,34(8,54)$ & & \\
\hline Europeu (no espanyol) & $24,6(6,23)$ & & \\
\hline Llatinoamericà & $33(10,13)$ & & \\
\hline Estat civil & & 0,742 & \\
\hline Fadrí/na o sense parella estable & $26,32(8,61)$ & & \\
\hline Casat /a o parella estable & $24,81(8,78)$ & & \\
\hline Curs & & & 0,479 \\
\hline $1 \mathrm{r}$ & $24,84(9,08)$ & & \\
\hline $2 n$ & $25,47(7,33)$ & & \\
\hline $3 r$ & $27,89(8,8)$ & & \\
\hline $4 \mathrm{t}$ & $24,87(8,77)$ & & \\
\hline Situació laboral & & 0,556 & \\
\hline Sols estudien & $26,58(8,7)$ & & \\
\hline Treball + estudis & $21,5(4,95)$ & & \\
\hline Assignatures pendents & & & 0,893 \\
\hline No & $24,85(8,61)$ & & \\
\hline Per suspens & $28,05(8,43)$ & & \\
\hline Per trasllat & $26,5(7,78)$ & & \\
\hline
\end{tabular}


Les diferències en funció de l'estat de salut informat, l'orientació sexual i la satisfacció amb la imatge corporal es recullen a la taula 4.

S'ha trobat que percebre un estat de salut física bo o molt bo, es relaciona amb menors puntuacions en estrès que si es percep com a dolent $(F=5,77 ; p=0,001)$, ja que les puntuacions en els dos primers casos són de 24,06 i 20,8 respectivament i en l'últim és de 37,4 .

Taula 4

Diferències en estrès segons salut, orientació sexual i satisfacció corporal

\begin{tabular}{|c|c|c|c|}
\hline Variable & $\begin{array}{c}\text { PSS14 } \\
\text { Mitja (DT) }\end{array}$ & $\mathrm{t}$ Student & $\begin{array}{c}\text { F } \\
\text { (Scheffé) }\end{array}$ \\
\hline Salut física & & & $5,77 * * *$ \\
\hline Molt bona & $20,8(10,33)$ & & $(\mathrm{D}>\mathrm{B}, \mathrm{MB})$ \\
\hline Bona & $24,06(7,91)$ & & \\
\hline Regular & $28,21(8,32)$ & & \\
\hline Dolenta & $37,4(2,41)$ & & \\
\hline Molt dolenta & - & & \\
\hline Malaltia física & & 0,004 & \\
\hline Sí & $25,82(9,48)$ & & \\
\hline No & $25,83(8,49)$ & & \\
\hline Salut mental & & & $13,95 * * *$ \\
\hline Molt bona & $16,82(8,46)$ & & $(\mathrm{D}, \mathrm{R}>\mathrm{B}>\mathrm{MB})$ \\
\hline Bona & $24,13(6,87)$ & & \\
\hline Regular & $31,55(6,54)$ & & \\
\hline Dolenta & $35(8,64)$ & & \\
\hline Molt dolenta & - & & \\
\hline Malaltia mental & & $2,063^{*}$ & \\
\hline Sí & $31,33(10,3)$ & & \\
\hline No & $25,16(8,25)$ & & \\
\hline Atracció sexual & & & 1,389 \\
\hline Només sexe oposat & $24,65(7,8)$ & & \\
\hline Principalment sexe oposat & $26,05(7,78)$ & & \\
\hline Un poc més sexe oposat & $28,3(10,08)$ & & \\
\hline Ambdós sexes per igual & $24,83(13,38)$ & & \\
\hline Un poc més mateix sexe & $36,5(0,71)$ & & \\
\hline Principalment mateix sexe & $27,75(6,55)$ & & \\
\hline Només mateix sexe & $15(2,83)$ & & \\
\hline Satisfacció corporal & & & $3,757 * *$ \\
\hline Gens & $34(8,87)$ & & \\
\hline Poca & $28,19(8,47)$ & & \\
\hline Bastant & $23,5(7,77)$ & & \\
\hline Molta & $22,14(8,57)$ & & \\
\hline
\end{tabular}

Nota: $\mathrm{MB}$, Molt bona; $\mathrm{B}$, bona; $\mathrm{R}$, regular; $\mathrm{D}$, dolenta $\mathrm{MD}$, molt dolenta $* \mathrm{p} \leq 0,05 ; * * \mathrm{p} \leq 0,01 ; * * * \mathrm{p} \leq 0,001$ 
De forma similar, percebre que l'estat de salut mental és dolent o regular (amb mitges en estrès de 35 i 31,55 respectivament) es relaciona amb major estrès que percebre'l com a bo (la mitja del qual és 24,13 ) i aquest també amb majors puntuacions que en les persones que informen d'una salut mental molt bona, i que de mitja obtenen 16,82 punts al PSS14.

En aquesta línia, les persones amb una malaltia mental diagnosticada puntuen de forma més alta en estrès (puntuació mitjana 31,33) que les que no en pateixen (puntuació mitjana 25,16), de forma estadísticament significativa (t=2,063; $p=0,042)$. També s'han donat diferències estadísticament significatives en allò que respecta a la satisfacció amb la imatge corporal $(F=3,757 ; p=0,010)$, variable en la qual es pot apreciar una menor puntuació en estrès a mesura que s'està més satisfet/a. No es van observar diferències estadísticament significatives quant a estrès percebut segons la presència o no de malaltia física diagnosticada ni en funció de l'atracció sexual.

\section{Discussió i conclusions}

Els resultats d'estrès percebut obtinguts han fet patent que el nivell d'estrès percebut és, per una part, molt elevat, ja que la prevalença d'estrès moderat-elevat es situa al voltant del $70 \%$ en la Facultat (és concretament del $72,61 \%$ en l'estudiantat de Psicologia), i, d'altra banda, que no és diferent entre titulacions de ciències de la salut. Aquestes dades recolzen les elevades prevalences descrites per investigacions prèvies (Merlo, Curran i Watson 2017; Pierceall i Keim 2007).

D'altra banda, hem pogut enumerar alguns dels estressors més prevalents en estudiants de Psicologia, de forma innovadora i que corresponen a la pèrdua del control del propi horari i els problemes econòmics o amb les relacions interpersonals principalment. Podem veure, no obstant això, que són de nou molt semblants als que la literatura existent descrivia per a l'estudiantat de Medicina.

Quant als factors associats a les majors puntuacions d'estrès, al Grau de Psicologia de I'UJI, aquestes es donen en aquelles persones que perceben un pitjor estat de salut tant física com mental, que pateixen una malaltia mental de base i estan més insatisfetes amb el seu cos, independentment d'altres característiques com el gènere, la situació laboral, el curs, haver de compaginar assignatures d'altres cursos, l'origen, o tindre parella. Així, juguen un paper més rellevant les cognicions sobre la salut i la imatge corporal que altres tipus de situacions, donant a entendre que el fet de conviure amb una malaltia mental, o no sentir-se plenament sà tant en el plànol físic com mental, és un estressor suficientment potent per desenvolupar nivells moderats $i$ alts d'estrès i de forma pareguda actuaria la insatisfacció amb la imatge corporal. Aquestes observacions són similars a les fetes en estudis precedents (Voltmer, Kötter i Spahn 2012) que relacionaren patrons d'experiències i conductes al treball de tipus saludable amb menor estrès i millor salut física, sobre una mostra d'estudiantat de Medicina.

Podem concloure que existeix una elevada proporció d'estudiantat de Psicologia amb nivells preocupants d'estrès, que es deuen fonamentalment a les percepcions particulars sobre l'estat de salut i la seua satisfacció. A més, els principals estressors detectats han estat de tipus organitzatiu (no controlar el propi horari, problemes econòmics).

Els nostres resultats, però, han de ser presos amb cura i necessiten ser replicats, donat el baix nombre de participants, a més de ser important descriure altres fonts de variabilitat quant a l'estrès $\mathrm{i}$ dissenys que permeten establir relacions de causalitat a 
diferència del present treball. Serien interesants futures línies d'investigació que posen l'èmfasi en els estils d'afrontament i variables de personalitat o que llancen llum sobre estratègies d'intervenció.

\section{Referències bibliogràfiques}

Admi, Hanna, Yahel Moshe-Eilon, Dganit Sharon i Michal Mann. 2018. «Nursing students' stress and satisfaction in clinical practice along different stages: A cross-sectional study». Nurse Education Today, 68: 86-92. https://doi.org/10.1016/j.nedt.2018.05.027

Bacchi, Stephen i Julio Licinio. 2017. «Resilience and Psychological Distress in Psychology and Medical Students». Academic Psychiatry, 41(2): 185-188. https://doi.org/10.1007/s40596-016-0488-0

Bore, Miles, Brian Kelly i Balakrishnan Nair. 2016. «Potential predictors of psychological distress and well-being in medical students: a cross-sectional pilot study». Advances in Medical Education and Practice, 7: 125-135. https://doi.org/10.2147/AMEP.S96802

Cohen, Sheldon, Tom Kamarck i Robin Mermelstein. 1983. «A global measure of perceived stress. Journal of Health and Social Behavior». Journal of Health and Social Behavior, 24(4), 385-396.

Dahlin, Marie, Nils Joneborg i Bo Runeson. 2005. "Stress and depression among medical students: a cross-sectional study». Medical Education, 39(6): 594-604. https://doi.org/10.1111/j.1365-2929.2005.02176.x

Dahlin, Marie i Bo Runeson. 2007. «Burnout and psychiatric morbidity among medical students entering clinical training: a three year prospective questionnaire and interview-based study». BMC Medical Education, 7(1): 6. https://doi.org/10.1186/1472-6920-7-6

Goel, Akhil, Sulbha Akarte, Sumita Agrawal i Vikas Yadav. 2016. «Longitudinal assessment of depression, stress, and burnout in medical students». Journal of Neurosciences in Rural Practice, 7(4): 493-498. https://doi.org/10.4103/09763147.188625

Guthrie, Else, Diamonique Black, Hermant Bagalkote, Chris Shaw, M. Campbell i Francis Creed. 1998. «Psychological stress and burnout in medical students: a five-year prospective longitudinal study». Journal of the Royal Society of Medicine, 91: 237-243.

Guthrie, Else, Diamonique Black, Chris Shaw, J. Hamilton, Francis Creed i Barbara Tomenson. 1997. "Psychological stress in medical students: A comparison of two very different university courses». Stress Medicine, 13(3): 179-184. https://doi.org/https://doi.org/10.1002/(SICI)1099-1700(199707)13:3<179::AIDSMI740>3.0.CO;2-E

Kötter, Thomas, Josefin Wagner, Linda Brüheim i Edgar Voltmer. 2017. «Perceived Medical School stress of undergraduate medical students predicts academic performance: An observational study». BMC Medical Education, 17(1): 1-6. https://doi.org/10.1186/s12909-017-1091-0

Matheson, Katherine, Tessa Barrett, Jeff Landine, Alan McLuckie, Nerissa Li Weh Soh i Garry Walter. 2016. «Experiences of Psychological Distress and Sources of Stress and Support during Medical Training: A Survey of Medical Students». Academic Psychiatry, 40(1): 63-68. https://doi.org/10.1007/s40596-015-0395-9

Merlo, Lisa, John Curran i Robert Watson. 2017. "Gender differences in substance use and psychiatric distress among medical students: A comprehensive statewide evaluation». Substance Abuse, 38(4): 401-406. https://doi.org/10.1080/08897077.2017.1355871 
Pierceall, Emily i Marybelle Keim. 2007. «Stress and coping strategies among community college students». Community College Journal of Research and Practice, 31(9): 703-712. https://doi.org/10.1080/10668920600866579

Sheets, Kent, Daniel Gorenflo i Mary Ann Forney. 1993. "Personal and Behavioral Variables Related to Perceived Stress of Second-Year Medical Students». Teaching and Learning in Medicine, 5(2): 90-95. https://doi.org/10.1080/10401339309539598

Suárez-García, José María, Alba Maestro-González, David Zuazua-Rico, Marta Sánchez-Zaballos i María Pilar Mosteiro-Díaz. 2018. «Stressors for Spanish nursing students in clinical practice». Nurse Education Today, 64: 16-20. https://doi.org/10.1016/j.nedt.2018.02.001

Valero-Chillerón, María Jesús, Víctor Manuel González-Chordá, Natividad LópezPeña, Águeda Cervera-Gasch, María Pilar Suárez-Alcázar i Desirée MenaTudela. 2019. "Burnout syndrome in nursing students: An observational study». Nurse Education Today, 76: 38-43. https://doi.org/10.1016/j.nedt.2019.01.014

Voltmer, Edgar, Thomas Kötter i Claudia Spahn. 2012. «Perceived medical school stress and the development of behavior and experience patterns in German medical students». Medical Teacher, 34(10): 840-847. https://doi.org/10.3109/0142159X.2012.706339

Youssef, Farid. 2016. «Medical Student Stress, Burnout and Depression in Trinidad and Tobago». Academic Psychiatry, 40(1): 69-75. https://doi.org/10.1007/s40596-015-0468-9

Zupiria Gorostidi, Xabier, Mari Jose Uranga Iturriotz, Mari Jose Alberdi Erize i Maite Barandiaran Lasa. 2003. "KEZKAK: cuestionario bilingüe de estresores de los estudiantes de enfermería en las prácticas clínicas». Gaceta Sanitaria, 17(1): 37-51. Recuperado de: http://scielo.isciii.es/pdf/gs/v17n1/orig5.pdf 